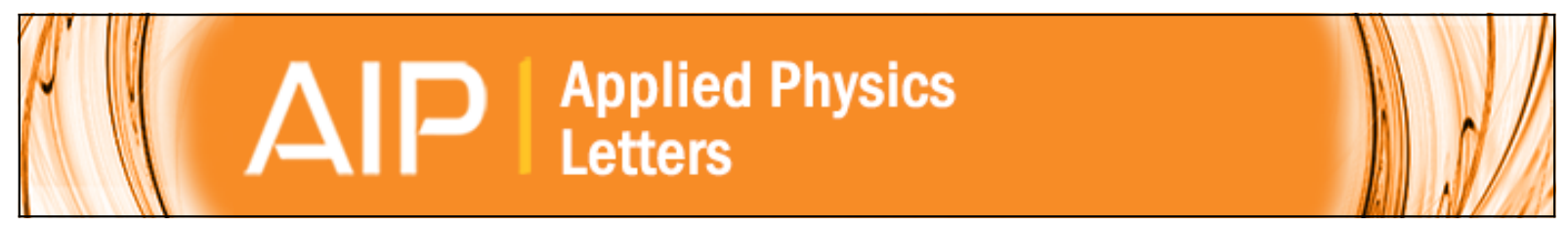

\title{
High-resolution Raman microscopy of curled carbon nanotubes
}

Hyunhyub Ko, Yuri Pikus, Chaoyang Jiang, Andrea Jauss, Olaf Hollricher, and Vladimir V. Tsukruk

Citation: Applied Physics Letters 85, 2598 (2004); doi: 10.1063/1.1795981

View online: http://dx.doi.org/10.1063/1.1795981

View Table of Contents: http://scitation.aip.org/content/aip/journal/apl/85/13?ver=pdfcov

Published by the AIP Publishing

\section{Articles you may be interested in}

Reinforcing multiwall carbon nanotubes by electron beam irradiation

J. Appl. Phys. 108, 084314 (2010); 10.1063/1.3493049

High resolution Raman imaging of single wall carbon nanotubes using electrochemically etched gold tips and a radially polarized annular beam

J. Vac. Sci. Technol. A 28, 472 (2010); 10.1116/1.3368499

Calibrating the single-wall carbon nanotube resonance Raman intensity by high resolution transmission electron microscopy for a spectroscopy-based diameter distribution determination

Appl. Phys. Lett. 96, 051910 (2010); 10.1063/1.3297904

Raman scattering test of single-wall carbon nanotube composites

Appl. Phys. Lett. 78, 3193 (2001); 10.1063/1.1373405

Effects of thermal annealing on the microstructure and mechanical properties of carbon-nitrogen films deposited by radio frequency-magnetron sputtering

J. Vac. Sci. Technol. A 17, 2811 (1999); 10.1116/1.582021

\section{AIP $\mid$ chaos \\ CALL FOR APPLICANTS Seeking new Editor-in-Chief}




\title{
High-resolution Raman microscopy of curled carbon nanotubes
}

\author{
Hyunhyub Ko, Yuri Pikus, and Chaoyang Jiang \\ Department of Materials Science \& Engineering, Iowa State University, Ames, Iowa 50011 \\ Andrea Jauss and Olaf Hollricher \\ WITec GmbH, Hoervelsinger Weg 6, 89081 Ulm, Germany \\ Vladimir V. Tsukruk ${ }^{\text {a) }}$ \\ Department of Materials Science \& Engineering, Iowa State University, Ames, Iowa 50011
}

(Received 29 April 2004; accepted 30 July 2004)

\begin{abstract}
Patterned carbon nanotube assemblies with bent nanotube bundles were investigated with combined atomic force microscopy and confocal Raman imaging spectroscopy to identify conditions of carbon nanotubes in the bent state. We showed that the tangential $G$ mode on Raman spectra systematically shifts downward upon nanotube bending as was predicted earlier. This lower frequency shift is attributed to the tensile stress, which results in the loosening of $\mathrm{C}-\mathrm{C}$ bonds in the outer nanotube walls. () 2004 American Institute of Physics. [DOI: 10.1063/1.1795981]
\end{abstract}

Carbon nanotubes (CNTs) are considered as prospective candidates in the development of CNT-based nanodevices due to a combination of their unique structural and physical properties related to electrical conductivity, chemical and thermal stability, high tensile strength, and elasticity. ${ }^{1,2}$ One essential prerequisite for the CNT-based nanodevices is controlling the shape, location, and orientation of large arrays of CNTs. A number of assembling methods have been proposed for the fabrication of such arrays including surface initiated growth and selective surface adsorption. ${ }^{3-9}$

The CNT assemblies anchored to chemically modified surfaces are likely to be under different mechanical stresses caused by a combination of capillary and surface forces resulting in their stretching, twisting, and bending. ${ }^{10-12} \mathrm{Re}-$ cently, it was reported that the mechanical deformation of CNTs strongly affects their electronic properties. ${ }^{13,14}$ It has been demonstrated that the mechanical strain can open a band gap in a metallic CNT and modify the band gap in a semiconducting CNT. ${ }^{13}$ Confocal Raman imaging and spectroscopy have shown that CNT structure may change along the tube axis possibly due to external stress or local defects in the tube structure. ${ }^{15-17}$ However, very little is revealed on the related optical properties of stressed nanotubes and direct acquisition for individual CNTs and their bundles under stress conditions was not demonstrated.

The relation between the Raman spectra and the basic structure of carbon nanotubes are intensely investigated and the nature of all major adsorption bands is well understood. ${ }^{18}$ For CNT composites, it was reported that compressive strain can induce frequency shift of Raman $G$ modes of SWNTs/ epoxy composites subjected to bending. ${ }^{19}$ However, these studies relied on the measurements of bulk CNT materials and their composites in which the transfer of mechanical stresses and actual shape of the stressed CNTs remain unknown to great extent. Raman studies of bulk CNT materials demonstrated frequency upshift with increasing compressive strain under hydrostatic pressure. ${ }^{20,21}$

In this study, we use a direct collection of Raman spectra from bent CNT bundles with precise localization of the probed area as monitored with combined atomic force mi-

\footnotetext{
${ }^{a)}$ Electronic mail: vladimir@iastate.edu
}

croscopy (AFM), confocal Raman microscopy, and high resolution micro-Raman spectroscopy. We used the surface organized arrays of bent and looped CNTs assembled by the contact line instabilities of patterned microfluidic flow as reported in our recent paper $^{8}$ and focused on finding characteristic Raman signature for bent arrays.

The experimental procedure for the patterned nanotube assembly is described in detail elsewhere. ${ }^{8,9}$ Raman mapping of the surface arrays and Raman spectroscopy from selected areas were conducted with two approaches. First, we used a confocal Raman microscope CRM 200 (WITec, Germany) with $532 \mathrm{~nm}$ line of a $\mathrm{Nd}$ : Yag laser for excitation. Avalanche photodiode detector in single photon counting mode was used for fast imaging. Alternatively, custom designed combined AFM-Raman setup based on near-field scanning optical microscope Aurora-3 (Digital Instruments) was used for Raman mapping and high-resolution Raman spectroscopy from selected locations. This setup was designed to be used for concurrent acquisition of surface topography in shear-force AFM mode and Raman images in a confocal scanning mode from beneath of the flat glass substrate. Excitation light from a $\mathrm{Nd}$ : Yag laser (cw second harmonic, $532 \mathrm{~nm}$ ) passes through controllable attenuator and beam expander, and after reflecting off a 50:50 beam splitter is fed into the Aurora-3 microscope. It is focused onto the sample surface by a high numerical aperture microscope objective $(40 \times / 0.65 \mathrm{NA}$ with a lateral resolution of $<0.5 \mu \mathrm{m})$. The signal collected from same objective passes through a notch filter and is focused on the entrance slit of imaging spectrograph SpectraPro SP-2558-W (Roper Scientific). CCD camera Spec-10:2KB (Roper Scientific) collects the spectra at every point of the sample. High-resolution spectra $\left(0.32 \mathrm{~cm}^{-1} /\right.$ pixel for $1800 \mathrm{~mm}^{-1}$ grating) can be obtained for the selected surface locations.

Figure 1(a) shows an AFM image of CNT patterned assembly with dense layer of CNT bundles of 10-20 nm height formed along amine-terminated stripes. ${ }^{9}$ The corresponding Raman $G$-mode image obtained by integrating the intensity of Raman spectra at $1590 \mathrm{~cm}^{-1}$ showed a close correlation with CNT pattern confirming preferential location of carbon nanotubes along the stripes [Fig. 1(b)]. Raman imaging clearly identifies the highly packed CNT assembly on 

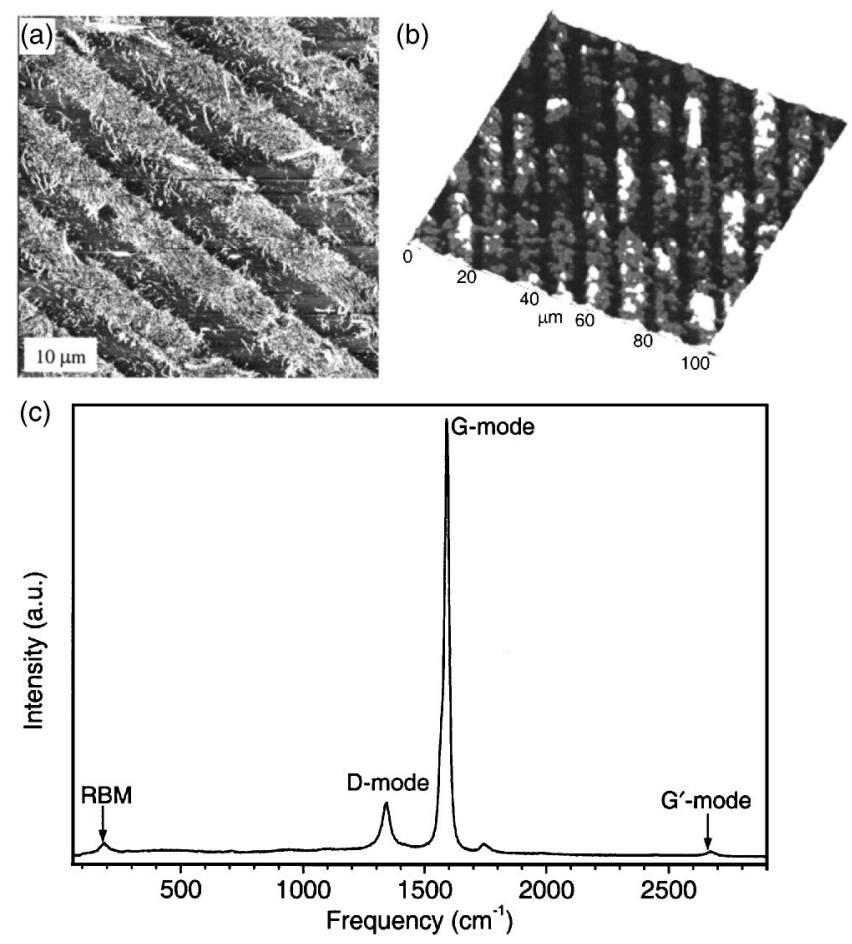

FIG. 1. (a) AFM topographical image of densely packed patterned array of carbon nanotubes. $Z$ range is $80 \mathrm{~nm}$. (b) Raman $G$ mode image of patterned nanotube assembly obtained by integrating the Raman intensity at $1590 \mathrm{~cm}^{-1}$. The Raman intensity varies from dark (low) to bright (high). (c) Raman spectrum of patterned nanotube arrays studied, showing the RBM, the $D$ mode, the $G$ mode, and $G^{\prime}$ mode.

patterned surface with variable intensity along the stripes reflecting dense CNT texture. ${ }^{9}$ Raman spectra taken on this sample show typical features of carbon nanotubes with radial breathing mode near $190 \mathrm{~cm}^{-1}, D$-mode near $1340 \mathrm{~cm}^{-1}, G$ mode near $1590 \mathrm{~cm}^{-1}$, and $G^{\prime}$ mode near $2670 \mathrm{~cm}^{-1}{ }^{22}$ The Raman combination mode near $1740 \mathrm{~cm}^{-1}$ is also observed. ${ }^{23}$ In this study, we focus on the strong Raman $G$ mode because the $G$ mode is attributed to tangential $\mathrm{C}-\mathrm{C}$ bond stretching mode, ${ }^{18}$ which should be affected by the local structural changes in carbon nanotubes and can be detected even for individual bundles.

The highly bent nanotube bundles in nest-shaped assemblies prepared according to the procedure reported earlier ${ }^{8,9}$ were studied with combined AFM and confocal Raman imaging and spectroscopy techniques (Fig. 2). The radius of nanotube "nest" was about $2 \mu \mathrm{m}$ and most of the nanotubes on the rim of $15-20 \mathrm{~nm}$ high are bent along the circumference of square-patterned areas and the straight nanotubes are concentrated in the center as confirmed by independent AFM scanning [Figs. 2(a) and 2(b)]. Raman mapping showed the higher intensity of the $G$ mode line along the rim for all curled assemblies that simply reflects much higher density of nanotube packing along the rims [Fig. 2(d)]. However, the careful analysis of the $G$-line position revealed a systematic peak shift along the bent bundles with a darker color representing shift to a lower frequency [Figs. 2(c) and 2(f)]. The shift of $2-4 \mathrm{~cm}^{-1}$ was systematically observed in the course of both independent experiments and with both medium and high-resolution modes of mapping. The shift observed was higher than natural deviations (usually within $\pm 1 \mathrm{~cm}^{-1}$ ) observed at different surface locations of the same specimen collected under identical experimental conditions although
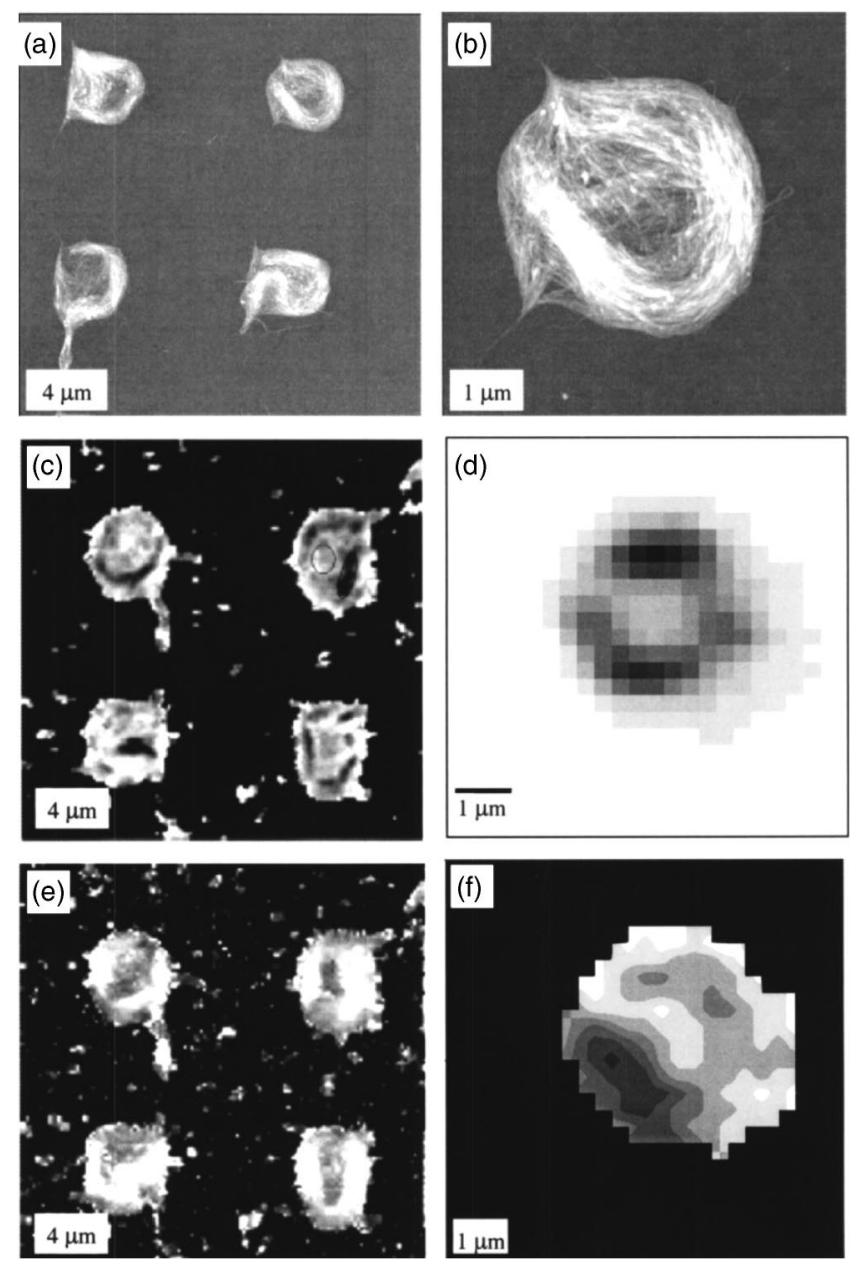

FIG. 2. (a) and (b) AFM topographical images of nest-shaped patterned nanotube assembly; (c) corresponding distribution of $G$-line position, varies from $1588 \mathrm{~cm}^{-1}$ (dark) to $1594 \mathrm{~cm}^{-1}$ (bright); (d) high resolution Raman image of the individual "nest" taken at $1594 \mathrm{~cm}^{-1}$, the intensity varies from light (low) to dark (high); (e) image of the linewidth (FWHM) of the $G$ mode, the linewidth varies from $16 \mathrm{~cm}^{-1}$ (dark) to $25 \mathrm{~cm}^{-1}$ (bright); (f) high resolution image of the individual "nest" taken at $G$ mode, position varies from $1591 \mathrm{~cm}^{-1}$ (bright) to $1594 \mathrm{~cm}^{-1}$ (dark).

larger deviations were observed from specimen to specimen. Moreover, this shift was accompanied by a significant (40\%-50\%) broadening of the $G$ line along the rims, a clear indication of increased local defects and the larger deviations in bundle-bundle interactions within arrays of curled nanotubes [Fig. 2(e)]..$^{24}$

Finally, we acquired concurrent AFM and Raman images from low density CNT arrays for the direct identification of the Raman spectrum with a specific portion of a nanotube bundle [Figs. 3(a) and 3(b)]. With this method, each CNT bundle identified within the white-dotted area in AFM image can be clearly resolved in sequentially obtained Raman maps [compare Figs. 3(a) and 3(b)]. Figure 3(c) shows the higher resolution Raman image of the selected curled bundle with two locations associated with straight and bent portions of the bundles. The Raman spectrum acquired from the bent portion ( $p 2)$ showed the lower frequency shift as compared to the straight portion of the bundle $(p 1)$. There is also a slight shift at lower energy shoulder on the $G$ line around $1570 \mathrm{~cm}^{-1}$ [Fig. 3(d)].

These changes in the Raman spectra for both curled nanotube arrays and individual bundles clearly indicate that 

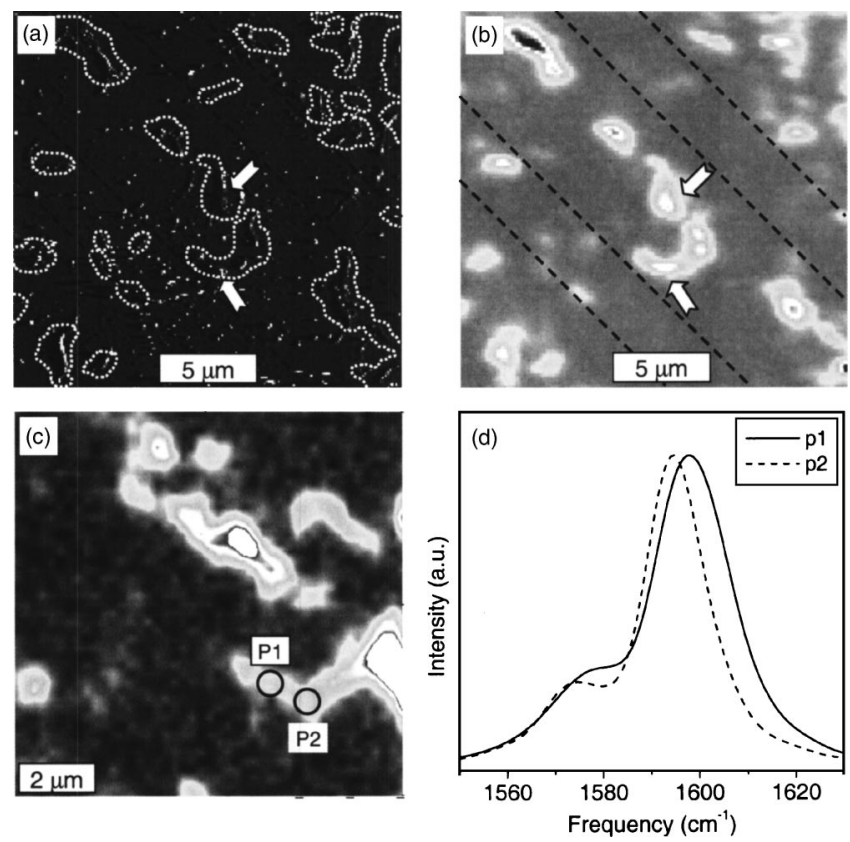

FIG. 3. Shear-force AFM (a) and Raman (b) images of low density nanotube arrays collected for the same surface area. The dashed lines show the patterned substrate and arrows indicate identical nanotubes. (c) High resolution Raman image of selected surface area. (d) Raman $G$ band recorded at two different positions as marked in Fig. 3(c).

there is a systematic shift in optical resonances brought by bent state of these nanotubes. ${ }^{15,17}$ Because of the nanotube bending and the corresponding shear stresses, ${ }^{12}$ the bent nanotubes are under combined tensile stress in the outer shell and compression stress in the inner wall. Considering only bending stress, the tensile strain $\left(\varepsilon_{t}\right)$ on the bent carbon nanotubes at the rim of the nest assemblies discussed here can be estimated as $\varepsilon_{t}=r / \rho$, where $r$ is tube radius and $\rho$ is radius of curvature. The estimation for $r=0.6 \mathrm{~nm}$ for individual carbon nanotubes with $\rho=1-2 \mu \mathrm{m}$ gives the tensile strain level of $\varepsilon_{t}=0.03 \%-0.06 \%$. The corresponding compression strain within the concave region of the bent nanotubes which is higher than the critical strain could result in nanotube buckling and complete compression stress relaxation. The critical compression strain value can be estimated from $\varepsilon_{c}=(\pi d / l)^{2} / 2$ where $d$ is the nanotube diameter and $l$ is the length of the bent section, which gives $\varepsilon_{c}=\sim 0.0002 \%$ for our conditions. ${ }^{25}$ We can conclude that the compression stress developed during bending causes buckling on the concave region of bent nanotubes and, thus, compression stress relaxation. This causes the uncompensated tensile stress in the outer radius of curled nanotubes to be dominating. In fact, buckling phenomenon of these curled nanotubes was confirmed by high resolution AFM imaging. ${ }^{9}$

Therefore, considering the buckling state of the bent nanotubes, we can associate the lower frequency shift in the $G$ mode observed here as the phenomenon caused by the presence of the high tensile stress in the outer shells of curled nanotube arrays. In fact, it has been suggested that the $G$ mode varies with the length of $\mathrm{C}-\mathrm{C}$ bond in carbon nanotube walls with a trend of lower frequency shift for the increasing length of these bonds. ${ }^{20-22}$ On the other hand, it has been observed that for carbon nanotubes under hydrostatic pressure, the compression strain results in shortening of $\mathrm{C}-\mathrm{C}$ bonds and corresponding shift of Raman $G$ peak to higher frequency.
In conclusion, we investigated the bent nanotube arrays by combined AFM and Raman mapping and spectroscopy and observed systematic lower frequency shift of the tangential $G$ mode. We suggested that the shift observed can be attributed to tensile strain of the bending nanotube arrays resulting in the loosening of $\mathrm{C}-\mathrm{C}$ bonds in the outer walls. We speculate that the shift in Raman spectra observed here can be used for the fast monitoring of the bending state of the standing carbon nanotube "forests" in gas and fluid flow nanosensors as well as for detecting stressed carbon nanotubes embedded into recently introduced free-suspended compliant nanomembranes for thermal and acoustic microsensing. ${ }^{26,27}$

This work is supported by NASA through NDE Center Contract No. NAG 102098, AFOSR, F496200210205, and AFOSR-DURIP F49620-03-1-0273 Grants.

${ }^{1}$ R. H. Baughman, A. A. Zakhidov, and W. A. de Heer, Science 297, 787 (2002).

${ }^{2}$ Carbon Nanotubes Synthesis Structure, Properties, and Applications, Topics in Applied Physics Vol. 80, edited by M. S. Dresslhaus, G. Dresselhaus, and P. Avouris (Springer, Heidelberg, 2000).

${ }^{3}$ S. Fan, M. Chapline, N. Franklin, T. Tombler, A. Cassell, and H. Dai, Science 283, 512 (1999).

${ }^{4}$ B. Q. Wei, R. Vajtai, Y. Jung, J. Ward, R. Zhang, G. Ramanath, and P. M. Ajayan, Nature (London) 416, 495 (2002).

${ }^{5}$ H. Shimoda, S. J. Oh, H. Z. Geng, R. J. Walker, X. B. Zhang, L. E. McNeil, and O. Zhou, Adv. Mater. (Weinheim, Ger.) 14, 899 (2002).

${ }^{6}$ S. J. Oh, Y. Cheng, J. Zhang, H. Shimoda, and O. Zhou, Appl. Phys. Lett. 82, 2521 (2003).

${ }^{7}$ S. G. Rao, L. Huang, W. Setyawan, and S. Hong, Nature (London) 425, 36 (2003).

${ }^{8}$ V. V. Tsukruk, H. Ko, and S. Peleshanko, Phys. Rev. Lett. 92, 065502 (2004).

${ }^{9}$ H. Ko, S. Peleshanko, and V. V. Tsukruk, J. Phys. Chem. B 108, 4385 (2004).

${ }^{10}$ W. Clauss, D. J. Bergeron, and A. T. Johnson, Phys. Rev. B 58, R4266 (1998).

${ }^{11}$ T. Hertel, R. E. Walkup, and P. Avouris, Phys. Rev. B 58, 13870 (1998).

${ }^{12}$ J.-P. Salvetat, G. A. D. Briggs, J.-M. Bonard, R. R. Bacsa, A. J. Kulik, T. Stockli, N. A. Burnham, and L. Forro, Phys. Rev. Lett. 82, 944 (1999).

${ }^{13}$ E. D. Minot, Y. Yaish, V. Sazonova, J.-Y Park, M. Brink, and P. L. McEuen, Phys. Rev. Lett. 90, 156401 (2003).

${ }^{14}$ A. Maiti, Nat. Mater. 2, 440 (2003).

${ }^{15}$ A. Mews, F. Koberling, T. Basché, G. Philipp, G. S. Duesberg, S. Roth, and M. Burghard, Adv. Mater. (Weinheim, Ger.) 12, 1210 (2000).

${ }^{16}$ A. Hartschuh, E. J. Sanchez, X. S. Xie, and L. Novotny, Phys. Rev. Lett. 90, 095503 (2003).

${ }^{17}$ C. Jiang, J. Zhao, H. A. Therese, M. Friedrich, and A. Mews, J. Phys. Chem. B 107, 8742 (2003).

${ }^{18}$ M. S. Dresselhaus and P. C. Ekiund, Adv. Phys. 49, 705 (2000).

${ }^{19}$ V. G. Hadjiev, M. N. Iliev, S. Arepalli, P. Nikolaev, and B. S. Files, Appl. Phys. Lett. 78, 3193 (2001).

${ }^{20}$ U. D. Venkateswaram, Phys. Rev. B 59, 10928 (1999).

${ }^{21}$ J. Sandier, M. S. P. Shaffer, A. H. Windle, M. P. Halsall, M. A. MontesMoran, C. A. Cooper, and R. J. Young, Phys. Rev. B 67, 035417 (2003).

${ }^{22}$ A. Jorio, M. A. Pimenta, A. G. Filho, R. Saito, G. Dresselhaus, and M. S. Dresselhaus, New J. Phys. 5, 139.1 (2003).

${ }^{23}$ J. Zaho, C. Jiang, Y. Fan, M. Burghard, T. Basché, and A. Mews, Nano Lett. 2, 823 (2002).

${ }^{24}$ A. Jorio, C. Fantini, M. S. S. Dantas, M. A. Pimenta, A. G Souza Filho, Ge. G. Samsonidze, V. W. Brar, G. Dresselhaus, M. S. Dresselhaus, A. K. Swan, M. S. Unlu, B. B. Goldberg, and R. Saito, Phys. Rev. B 66, 115411 (2002).

${ }^{25}$ B. I. Yakobson, C. J. Brabec, and J. Bernholc, Phys. Rev. Lett. 76, 2511 (1996).

${ }^{26}$ C. Jiang, S. Markutsya, and V. V. Tsukruk, Adv. Mater. (Weinheim, Ger.) 16, 157 (2004).

${ }^{27}$ C. Jiang, S. Markutsya, and V. V. Tsukruk, Langmuir 20, 882 (2004). 